\title{
Comment on "Improvement of the distance between intuitionistic fuzzy sets and its applications"
}

\author{
Changlin $\mathrm{Xu}^{*}$ \\ School of Mathematics and Information Science, North Minzu University, Yinchuan, China
}

\begin{abstract}
Here, necessary corrections on the proof the Theorem 1 of Xu (J Intell Fuzzy Syst 33(3): 1563-1575, 2017) are stated in brief. Throughout, we use the same notations and equation numbers as in $\mathrm{Xu}$.
\end{abstract}

Keywords: Intuitionistic fuzzy sets, distance measure, Euclidean distance

Intuitionistic fuzzy sets(IFSs) were proposed by Atanassov [1] as a generalization of the fuzzy sets. As the most interesting topics in IFSs theory, distance measures are involved in fuzzy decision making, patter recognition, fuzzy reasoning, etc, [2-6].

In 2017, a measuring distance between intuitionistic fuzzy sets, proposed by $\mathrm{Xu}$ [5], was successfully applied into pattern recognition problems and medical diagnosis. However, there is a small mistake about the proof of the Theorem 1 in Xu [5]. In order to show the detailed correction instructions, the definitions involved in the paper [5] are as follows.

Definition 1. A metric distance $D$ in a non-empty set $X$ is a real value function $D: X \times X \rightarrow[0,+\infty)$, which satisfies the following conditions, $\forall x, y, z \in X$ :

(MD1) $D(x, y)=0$ if and only if $x=y$;

(MD2) $D(x, y)=D(y, x)$;

(MD3) $D(x, y)+D(y, z) \geq D(x, z)$.

Definition 2. [7] Let $D$ be a mapping: $\operatorname{IFSs}(X) \times$ $\operatorname{IFSs}(X) \rightarrow[0,1]$. For $\forall A, B, C \in \operatorname{IFSs}(X), D(A, B)$ is a distance measure between IFSs $A$ and $B$, if $D$ satisfies the following properties:

${ }^{*}$ Corresponding author. Changlin $\mathrm{Xu}$, School of Mathematics and Information Science, North Minzu University, Yinchuan 750021, China. E-mail: xu_changlin@nun.edu.cn.

$$
\begin{aligned}
& (D P 1) 0 \leq D(A, B) \leq 1 ; \\
& (D P 2) D(A, B)=0 \text { if and only if } A=B ; \\
& (D P 3) D(A, B)=D(B, A) ; \\
& (D P 4) \text { If } A \subseteq B \subseteq C \text {, then } D(A, C) \geq D(A, B), \\
& D(A, C) \geq D(B, C) .
\end{aligned}
$$

Definition 3. [5] Let $A=\left\{\left\langle x, \mu_{A}(x), v_{A}(x)\right\rangle \mid x \in X\right\}$ be a IFSs in $X=\{x\}$, then the assignments of the hesitancy degree $\pi_{A}(x)$ to membership degree $\mu_{A}(x)$ and nonmembership degree $v_{A}(x)$ are defined as

$$
\begin{aligned}
\operatorname{Assign}_{A}^{\pi \mu}(x) & =\left[\pi_{A}(x)+2 \mu_{A}(x)\right] / 2, \\
\operatorname{Assign}_{A}^{\pi \nu}(x) & =\left[\pi_{A}(x)+2 v_{A}(x)\right] / 2 .
\end{aligned}
$$

We take the four parts $\mu_{A}(x), v_{A}(x), \operatorname{Assign}_{A}^{\pi \mu}(x)$ and $\operatorname{Assign}_{A}^{\pi \nu}(x)$ into account the distances between IFSs, thereby a new distance measure, denoted as $D_{\text {IFSs }}$, is defined.

Definition 4. [5] Let $A=\left\{\left\langle x, \mu_{A}(x), v_{A}(x)\right\rangle \mid x \in\right.$ $X\}, B=\left\{\left\langle x, \mu_{B}(x), v_{B}(x)\right\rangle \mid x \in X\right\}$ be two IFSs in $X=\{x\}$, then the distance measure between $A$ and $B$ is defined as

$$
\begin{aligned}
& D_{\mathrm{IFSs}}(A, B)= \\
& \quad \frac{1}{2} \sqrt{\left(\Delta_{\mu}^{A B}\right)^{2}+\left(\Delta_{v}^{A B}\right)^{2}+\left(\Delta_{\pi \mu}^{A B}\right)^{2}+\left(\Delta_{\pi \nu}^{A B}\right)^{2}},
\end{aligned}
$$


where, $\quad \Delta_{\mu}^{A B}=\mu_{A}-\mu_{B}, \quad \Delta_{v}^{A B}=v_{A} v_{B}, \quad \Delta_{\pi \mu}^{A B}=$ $\operatorname{Assign}_{A}^{\pi \mu}-\operatorname{Assign}_{B}^{\pi \mu}$ and $\Delta_{\pi \nu}^{A B}=\operatorname{Assign}_{A}^{\pi \nu}-\operatorname{Assign}_{B}^{\pi \nu}$.

Theorem 1. [5] Let $A=\left\{\left\langle x, \mu_{A}(x), v_{A}(x)\right\rangle \mid x \in X\right\}$, $B=\left\{\left\langle x, \mu_{B}(x), v_{B}(x)\right\rangle \mid x \in X\right\}$ be two IFSs in $X=\{x\}$, then $D_{\mathrm{IFSs}}(A, B)$ is a distance measure satisfying the Definition 1 and Definition 2.

In the paper [5], the proof of the third step is as follows.

3) For $\forall A, B, C \in \operatorname{IFSs}(X)$, we have

$$
\begin{aligned}
& \left(\Delta_{\mu}^{A C}\right)^{2}=\left(\Delta_{\mu}^{A B}+\Delta_{\mu}^{B C}\right)^{2} \leq\left(\Delta_{\mu}^{A B}\right)^{2}+\left(\Delta_{\mu}^{B C}\right)^{2} ; \\
& \left(\Delta_{v}^{A C}\right)^{2}=\left(\Delta_{\nu}^{A B}+\Delta_{v}^{B C}\right)^{2} \leq\left(\Delta_{\nu}^{A B}\right)^{2}+\left(\Delta_{\nu}^{B C}\right)^{2} ; \\
& \left(\Delta_{\pi \mu}^{A C}\right)^{2}=\left(\Delta_{\pi \mu}^{A B}+\Delta_{\pi \mu}^{B C}\right)^{2} \leq\left(\Delta_{\pi \mu}^{A B}\right)^{2}+\left(\Delta_{\pi \mu}^{B C}\right)^{2} ; \\
& \left(\Delta_{\pi \nu}^{A C}\right)^{2}=\left(\Delta_{\pi \nu}^{A B}+\Delta_{\pi \nu}^{B C}\right)^{2} \leq\left(\Delta_{\pi \nu}^{A B}\right)^{2}+\left(\Delta_{\pi \nu}^{B C}\right)^{2} .
\end{aligned}
$$

Thus, $D_{\mathrm{IFSs}}(A, C) \leq D_{\mathrm{IFSs}}(A, B)+D_{\mathrm{IFSs}}(B, C)$, which indicates that $D_{\mathrm{IFSs}}$ satisfies $(M D 3)$.

However, the conclusion $D_{\mathrm{IFSs}}(A, C) \leq$ $D_{\mathrm{IFSs}}(A, B)+D_{\mathrm{IFSs}}(B, C)$ is derived from the formula (3), which is a wrong logical reasoning. Where, it should be noted that the formula (3) is correct. In fact, from the formula (3) and the property of inequality, we can obtained

$$
\begin{aligned}
& {\left[\left(\Delta_{\mu}^{A C}\right)^{2}+\left(\Delta_{\nu}^{A C}\right)^{2}+\left(\Delta_{\pi \mu}^{A C}\right)^{2}+\left(\Delta_{\pi v}^{A C}\right)^{2}\right] \leq} \\
& \left\{\left[\left(\Delta_{\mu}^{A B}\right)^{2}+\left(\Delta_{v}^{A B}\right)^{2}+\left(\Delta_{\pi \mu}^{A B}\right)^{2}+\left(\Delta_{\pi v}^{A B}\right)^{2}\right]\right. \\
& \left.+\left[\left(\Delta_{\mu}^{B C}\right)^{2}+\left(\Delta_{v}^{B C}\right)^{2}+\left(\Delta_{\pi \mu}^{B C}\right)^{2}+\left(\Delta_{\pi v}^{B C}\right)^{2}\right]\right\} .
\end{aligned}
$$

While, from the Definition 4, we have

$$
\begin{aligned}
& 4\left[D_{\mathrm{IFSs}}(A, C)\right]^{2}=\left(\Delta_{\mu}^{A C}\right)^{2}+\left(\Delta_{v}^{A C}\right)^{2}+\left(\Delta_{\pi \mu}^{A C}\right)^{2}+\left(\Delta_{\pi \nu}^{A C}\right)^{2}, \\
& 4\left[D_{\mathrm{IFSs}}(A, B)\right]^{2}=\left(\Delta_{\mu}^{A B}\right)^{2}+\left(\Delta_{v}^{A B}\right)^{2}+\left(\Delta_{\pi \mu}^{A B}\right)^{2}+\left(\Delta_{\pi \nu}^{A B}\right)^{2}, \\
& 4\left[D_{\mathrm{IFSs}}(B, C)\right]^{2}=\left(\Delta_{\mu}^{B C}\right)^{2}+\left(\Delta_{v}^{B C}\right)^{2}+\left(\Delta_{\pi \mu}^{B C}\right)^{2}+\left(\Delta_{\pi \nu}^{B C}\right)^{2} .
\end{aligned}
$$

Therefore,

$$
\left[D_{\mathrm{IFSs}}(A, C)\right]^{2} \leq\left[D_{\mathrm{IFSs}}(A, B)\right]^{2}+\left[D_{\mathrm{IFSs}}(B, C)\right]^{2} .
$$

However, based on the formula (5), it is not obtained

$$
D_{\mathrm{IFSs}}(A, C) \leq D_{\mathrm{IFSs}}(A, B)+D_{\mathrm{IFSs}}(B, C) .
$$

This shows that the proof of the paper [5] is incorrect.

The proper proof of the third step is as follows.

3) According to the Definition 4, the distance measure $2 D_{\mathrm{IFSs}}(A, B)$ can be viewed as the Euclidean distance between two points $\quad\left(\mu_{A}, v_{A}, \operatorname{Assign}_{A}^{\pi \mu}\right.$, Assign $\left._{A}^{\pi \nu}\right) \quad$ and $\left(\mu_{B}, v_{B}, \operatorname{Assign}_{B}^{\pi \mu}\right.$, Assign $\left.n_{B}^{\pi \nu}\right)$ in a four-dimensional real number space. For $\forall A, B, C \in \operatorname{IFSs}(X)$, there are three real points.

$$
\begin{aligned}
& A:\left(\mu_{A}, v_{A}, A_{s s i g n}^{\pi \mu}, \text { Assign }_{A}^{\pi \nu}\right), \\
& B:\left(\mu_{B}, v_{B}, A s s i g n_{B}^{\pi \mu}, \text { Assign } n_{B}^{\pi \nu}\right), \\
& C:\left(\mu_{C}, v_{C}, A s s i g n_{C}^{\pi \mu}, A s s i g n_{C}^{\pi \nu}\right) .
\end{aligned}
$$

Since the distance measure $2 D_{\mathrm{IFSs}}(A, B)$ is a metric distance, based on the third condition $(M D 3)$ of the Definition 1 (The properties of triangular inequalities for Euclidean distance), we have

$$
2 D_{\mathrm{IFSs}}(A, C) \leq 2 D_{\mathrm{IFSs}}(A, B)+2 D_{\mathrm{IFSs}}(B, C),
$$

which yields

$$
D_{\mathrm{IFSs}}(A, C) \leq D_{\mathrm{IFSs}}(A, B)+D_{\mathrm{IFSs}}(B, C) .
$$

The result shows that $D_{\text {IFSs }}$ satisfies (MD3).

\section{Acknowledgment}

The author would like to thank for a grant from the Ningxia Natural Science Foundation (No. 2018AAC03253), the First-Class Disciplines Foundation of Ningxia (No. NXYLXK2017B09), the key project of North Minzu University (No. ZDZX20 1801, ZDZX201804), the National Natural Science Foundation of China (No. 61662001, 11761002)

\section{References}

[1] K.T. Atanassov, Intuitionistic fuzzy sets, Fuzzy Sets Syst 20 (1986), 87-96.

[2] A.G. Hatzimichailidis, G.A. Papakostas and V.G. Kaburlasos, Anovel distance measure of intuitionistic fuzzy sets and its application to pattern recognition problems, Int J Intell Syst 27 (2012), 396-409.

[3] Y. Yang and F. Chiclana, Consistency of $2 \mathrm{~d}$ and $3 \mathrm{~d}$ distances of intuitionistic fuzzy sets, Expert Syst Appl 39 (2012), 8865-8670.

[4] Y.W. Du and W.M. Xu, Multiattribute group decision making based on interval-valued intuitionistic fuzzy sets and analytically evidential reasoning methodology, J Intell Fuzzy Syst 33 (2017), 2953-2960.

[5] C.L. Xu, Improvement of the distance between intuitionistic fuzzy sets and its applications, J Intell Fuzzy Syst 33 (2017), $1563-1575$.

[6] R. Joshi, S. Kumar, D. Gupta and H. Kaur, A Jensen-alphaNorm dissimilarity measure for intuitionistic fuzzy sets and its applications in multiple attribute decision making, Int $J$ Fuzzy Syst 20 (2018), 1188-1202.

[7] D. Wang and X. Xin, Distance measure between intuitionistic fuzzy sets, Pattern Recogn Lett 26 (2005), 2063-2069. 